\title{
CONTINUOUS EXTENSION OF ANOSOV FOLIATIONS IN 3-MANIFOLDS WITH NEGATIVELY CURVED FUNDAMENTAL GROUP
}

\author{
SÉRGIO R. FENLEY
}

\begin{abstract}
We study Anosov flows in 3-manifolds whose stable and unstable foliations in the universal cover have Hausdorff leaf space. We show that the intrinsic ideal boundaries of distinct stable leaves can be canonically identified and similarly for the unstable foliation. This is then applied to the case when the 3manifold has negatively curved fundamental group and leaves of the above foliations extend continuously to the ideal boundaries. We prove that the continuous extension restricted to the ideal boundaries respects the identifications of intrinsic ideal points mentioned above. We also analyse the non injectivity of the extension to the boundaries and show that there are uncountably many almost periodic, non periodic orbits of the flow which lift to flow lines with same ideal point in both directions. Finally we prove that the image of any open set in the domain ideal boundary, contains open sets in the range ideal boundary.
\end{abstract}

\section{Introduction and statements of results.}

The purpose of this article is twofold. First we want to explore the rich structure of R-covered Anosov flows in 3-manifolds (defined below) and second we study metric properties of these flows when the underlying manifolds have negatively curved fundamental group as defined by Gromov [Gr], here called ncl manifolds.

A codimension one foliation is said to be $\mathbf{R}$-covered if the leaf space of its lift to the universal cover is homeomorphic to the set of real numbers. An Anosov flow is $\mathbf{R}$-covered when one (equivalently both [Fe3]) of the stable or unstable foliations is $\mathbf{R}$-covered. For Anosov foliations this is equivalent to having Hausdorff leaf space and it implies that the flow is transitive [So, Ba1]. The flows in the two classical families of Anosov flows in dimension 3, namely, suspensions of Anosov diffeomorphisms of the torus $T^{2}$ (briefly suspensions) and geodesic flows in the unit tangent bundle of surfaces of negative curvature (briefly geodesic flows), are always $\mathbf{R}$-covered. There is also a large class of $\mathbf{R}$-covered Anosov flows in hyperbolic 3-manifolds, obtained for example by Dehn surgery on closed orbits of suspensions and 
geodesic flows $[\mathbf{G o}],[\mathbf{F e} 3]$ and there are also many examples in graph manifolds $[\mathbf{B a 2}]$. In fact, only very recently an example was found of a transitive, non R-covered Anosov flow in dimension 3 [Bo-La]. So far there are no such examples in ncl 3-manifolds.

Furthermore R-covered Anosov flows enjoy many good properties [Fe3]. In particular, the joint topological structure of $\widetilde{\mathcal{F}}^{s}, \widetilde{\mathcal{F}}^{u}$ can only be of two types, corresponding to the topological type of suspensions (product type) or of geodesic flows (skewed type), see definitions of product and skewed in Section 2. The product type is very simple, but the skewed type has a rich structure which will be explored here.

We first describe the intrinsic geometry of the leaves for a general Anosov flow. Let $\Phi$ be an Anosov flow in $M^{3}$ and let $\mathcal{F}^{s}, \mathcal{F}^{u}$ be the 2-dimensional (weak) stable and unstable foliations of $\Phi$. Let $\pi: \widetilde{M} \rightarrow M$ be the universal cover of $M$ and let $\widetilde{\Phi}, \widetilde{\mathcal{F}}^{s}, \widetilde{\mathcal{F}}^{u}$ be the respective lifts to $\widetilde{M}$. A leaf of $\widetilde{\mathcal{F}}^{s}$ or $\widetilde{\mathcal{F}}^{u}$ has an induced Riemannian metric from the universal cover $\widetilde{M}$ and in this metric, the leaf is negatively curved in the large in the Gromov sense [Su]. As a result it has an ideal boundary $[\mathbf{G r}]$ which is always homeomorphic to a circle $[\mathbf{F e} 1]$ and is denoted by $\partial_{\infty} F$. Furthermore $F \cup \partial_{\infty} F$ is homeomorphic to the closed disk. Flow lines of $\widetilde{\Phi}$ in $F$ are quasigeodesics in the intrinsic metric [Fe3], hence define two ideal points (forward and backward directions). Ideal points of $F$ correspond to the distinct negative limit points of flow lines and the common forward limit point of all flow lines - which are forward asymptotic.

All results here are valid for both $\mathcal{F}^{s}$ and $\mathcal{F}^{u}$, but for simplicity will be often be stated and proved only for the stable foliation.

Theorem A. Let $\Phi$ be an $\mathbf{R}$-covered Anosov flow in $M^{3}$ closed. Then for any $F, L \in \widetilde{\mathcal{F}}^{s}$, there is a canonical homeomorphism $X_{F}^{L}: \partial_{\infty} F \rightarrow \partial_{\infty} L$. Hence there is a "global" stable ideal boundary $S_{\infty}^{s}$ and a well defined map $\zeta_{F}: \partial_{\infty} F \rightarrow S_{\infty}^{s}$, so that for any $F, L \in \widetilde{\mathcal{F}}^{s}, \zeta_{F}=\zeta_{L} \circ X_{F}^{L}$.

The homeomorphism $X_{F}^{L}$ is invariant under covering translations of $\widetilde{M}$ in the following sense: Given a covering translation $g$ of $\widetilde{M}$ taking $F$ to $L$, then $g$ induces a homeomorphism $h_{1}: \partial_{\infty} F \rightarrow \partial_{\infty} L$. If $V$ is any leaf of $\widetilde{\mathcal{F}}^{s}$ and $E=g(V), h_{2}: \partial_{\infty} V \rightarrow \partial_{\infty} E$, then $h_{2} \circ X_{F}^{V}=X_{L}^{E} \circ h_{1}$.

The $\mathbf{R}$-covered hypothesis is essential for this result, for in general there is no natural way to relate ideal points in distinct leaves. We also show that ideal boundaries of leaves are naturally identified to a $\mathbf{Z}$ quotient of the leaf space of the dual foliation (the dual of the stable foliation is the unstable foliation and vice versa). This fact will be used in many arguments.

We now discuss metric properties. The two classical families have the best possible metric behavior: It is easy to see that, in the appropriate 
metrics, the leaves of the stable and unstable foliations lift to totally geodesic submanifolds in the universal cover and flow lines lift to minimal geodesics.

The metric behavior of Anosov flows in hyperbolic 3-manifolds is not so easy to determine. In these manifolds, instead of totally geodesic, it is better to consider the following (weaker) quasi properties [Th1]: A properly embedded submanifold of $\widetilde{M}$ is quasi-isometric if distance in the submanifold and distance in $\widetilde{M}$ are at most a bounded (multiplicative) distortion of each other. A foliation is quasi-isometric when lifts of all its leaves to the universal cover are quasi-isometric. The 1-dimensional case is called quasigeodesic.

Hyperbolic manifolds are always ncl $[\mathbf{G r}]$ and we will analyse metric properties in the class of ncl manifolds. In [Fe1] we proved that no codimension one foliation of an ncl 3-manifold can be quasi-isometric. Since Anosov flows in such manifolds are always transitive [Fe4], it follows that no stable or unstable leaf can be quasi-isometrically embedded. The quasigeodesic question for flow lines has only been settled in the $\mathbf{R}$-covered case: An $\mathbf{R}$-covered Anosov flow in an ncl 3-manifold can never be quasigeodesic [Fe3]. These results lead us to consider the weaker metric property, discussed below, of continuous extension of leaves to the ideal boundary.

If $M^{3}$ is ncl, then $\widetilde{M}$ can also be canonically compactified with an ideal boundary $[\mathbf{G r}]$ which is always homeomorphic to a 2-dimensional sphere [Be-Me] and is denoted by $S_{\infty}^{2}$. In addition $\widetilde{M} \cup S_{\infty}^{2}$ is homeomorphic to a closed 3-ball [Be-Me]. In addition the leaves of any codimension one foliation $\mathcal{F}$ of $M$ are always negatively curved in the large [Can]. The foliation $\widetilde{\mathcal{F}}$ is said to have the continuous extension property (briefly CEP) if for all $F \in \widetilde{\mathcal{F}}$, the inclusion $\varphi_{F}: F \rightarrow \widetilde{M}$ extends continuously to the ideal boundaries:

$$
\varphi_{F}: F \cup \partial_{\infty} F \rightarrow \widetilde{M} \cup S_{\infty}^{2}
$$

The restriction to ideal boundaries will be denoted by $\varphi_{F}^{\infty}: \partial_{\infty} F \rightarrow S_{\infty}^{2}$.

There are many cases where this happens. For instance if $\mathcal{F}$ is a fibration of $M$ over the circle, then CEP was proved by Cannon and Thurston [Ca-Th] and this property was also proved for many depth one foliations in hyperbolic 3-manifolds $[\mathbf{F e} 2]$. In fact, in these cases, the topological structure of the foliation produces a pseudometric in $M$. For instance in the case of fibration the input is any hyperbolic metric in the fiber, plus the homotopy class of the monodromy (which has to be a pseudo-Anosov homeomorphism [Th2]). The pseudo-Anosov map has stable and unstable measured foliations which are projectively invariant under the monodromy. This data produces a pseudo-metric in $M$ so that when lifted to the universal cover it is quasi-isometric to the hyperbolic metric in $\mathbf{H}^{3}[\mathbf{C a}-\mathbf{T h}]$. Notice that individual leaves are not quasi-isometrically embedded. 
The key tool in these cases was the existence of a compact leaf, whose geometric behavior is completely characterized as being either quasi-Fuchsian or a virtual fiber [Th1, Bon]. Using this one builds a hierarchy to understand all leaves. One big difficulty in studying Anosov foliations is that they do not have compact leaves. In fact in our situation, leaves are dense.

We start the study of this problem for Anosov foliations by assuming the continuous extension property and then analysing its consequences. We first show that the identification of intrinsic ideal points of leaves given by theorem $A$ is respected by the extension maps.

Theorem B. Let $\Phi$ be an $\mathbf{R}$-covered Anosov flow in $M^{3}$ with negatively curved fundamental group. Suppose that $\widetilde{\mathcal{F}}^{s}$ and $\widetilde{\mathcal{F}}^{u}$ have the continuous extension property. Then there is a continuous map $\varphi_{s}: S_{\infty}^{s} \rightarrow S_{\infty}^{2}$ so that for any $F \in \widetilde{\mathcal{F}}^{s}$

$$
\varphi_{F}^{\infty}=\varphi_{s} \circ \zeta_{F} .
$$

The same result holds for $\widetilde{\mathcal{F}}^{u}$.

We remark that we need to assume that both $\widetilde{\mathcal{F}}^{s}$ and $\widetilde{\mathcal{F}}^{u}$ have CEP to get the result in theorem $B$, even if we may be interested in only one of the foliations, say the stable one. We say that $\Phi$ has CEP if both $\widetilde{\mathcal{F}}^{s}$ and $\widetilde{\mathcal{F}}^{u}$ have CEP. Using this it is easy to prove:

Theorem C. Let $\Phi$ be an $\mathbf{R}$-covered Anosov flow in $M^{3}$ with negatively curved fundamental group so that $\Phi$ has the continuous extension property. For any open set $B \subset S_{\infty}^{s}, \varphi_{s}(B)$ contains an open set in $S_{\infty}^{2}$.

As will be clear from the proof we do not quite show $\varphi$ is an open map.

A periodic leaf $F$ of $\widetilde{\mathcal{F}}^{s} \cup \widetilde{\mathcal{F}}^{u}$ is one for which there is a non trivial covering translation $g$ of $\widetilde{M}$, so that $g(F)=F$. This occurs if and only if $\pi(F)$ contains a periodic orbit of $\Phi$.

The limit set of $F \subset \widetilde{M}$ is the set of accumulation points of $F$ in $S_{\infty}^{2}$ and is denoted by $\Lambda_{F}$. If $\Phi$ is $\mathbf{R}$-covered and $M$ is ncl, then $\Lambda_{F}$ is always equal to $S_{\infty}^{2}$, for any $F \in \widetilde{\mathcal{F}}^{s} \cup \widetilde{\mathcal{F}}^{u}[\mathbf{F e} 1]$. By topological considerations the map $\varphi_{s}$ cannot be injective. The next result is better stated in terms of the ideal boundary $\partial_{\infty} F$.

Theorem D. Let $\Phi$ be an $\mathbf{R}$-covered Anosov flow in $M^{3}$ with negatively curved fundamental group so that $\Phi$ has the continuous extension property. Let $F \in \widetilde{\mathcal{F}}^{s}$ and let $p \in \partial_{\infty} F$ which is the intrinsic negative ideal point of a flow line $\gamma$ of $\widetilde{\Phi}$, so that $\gamma$ is contained in a periodic unstable leaf $G \in \widetilde{\mathcal{F}}^{u}$. Then for any $q \in \partial_{\infty} F$, with $q \neq p$, it follows that $\varphi_{F}^{\infty}(p) \neq \varphi_{F}^{\infty}(q)$.

This is the opposite result from the quasigeodesic, non $\mathbf{R}$-covered case in $M^{3} \mathrm{ncl}[\mathbf{F e} 4]$. In that situation if 2 flow lines in the same stable leaf had 
the same ideal negative limit point in $S_{\infty}^{2}$, then both of them had to be in periodic unstable leaves, invariant under a common covering translation.

On the other hand, we show there are many orbits with same ideal points in both directions:

Theorem E. Let $\Phi$ be an $\mathbf{R}$-covered Anosov flow in $M^{3}$ ncl and assume that $\Phi$ has $C E P$. Then there are uncountably many almost periodic, non periodic orbits $\gamma$ of $\Phi$, so that any lift $\widetilde{\gamma}$ of $\gamma$ to $\widetilde{M}$ has the same ideal points in forward and backwards directions.

We thank the referee for a careful reading and detailed suggestions which greatly improved the presentation of this paper.

\section{Canonical homemorphisms between intrinsic ideal boundaries.}

General references for Anosov flows are $[\mathbf{A n}, \mathbf{S m}]$. Let $\Phi$ be an Anosov flow in $M^{3}$ (M ncl or $\Phi$ not assumed a priori) and let $\widetilde{\Phi}$ be the lift of $\Phi$ to $\widetilde{M}$. Then the orbit space of $\widetilde{\Phi}, \mathcal{O}=\widetilde{M} / \widetilde{\Phi}$, is homeomorphic to $\mathbf{R}^{2}[\mathbf{F e} 3$ ], a fact which helps in visualizing many arguments. Then $\widetilde{\mathcal{F}}^{s}, \widetilde{\mathcal{F}}^{u}$ induce transverse, 1-dimensional foliations in $\mathcal{O}$, denoted by the same symbols. Let $\mathcal{H}^{s}$ be the leaf space of $\widetilde{\mathcal{F}}^{s}$ and similarly define $\mathcal{H}^{u}$.

If $\Phi$ is also $\mathbf{R}$-covered, then up to isotopy there are only two topological models for the joint structure of the stable and unstable foliations in the universal cover [Fe3], which we now describe.
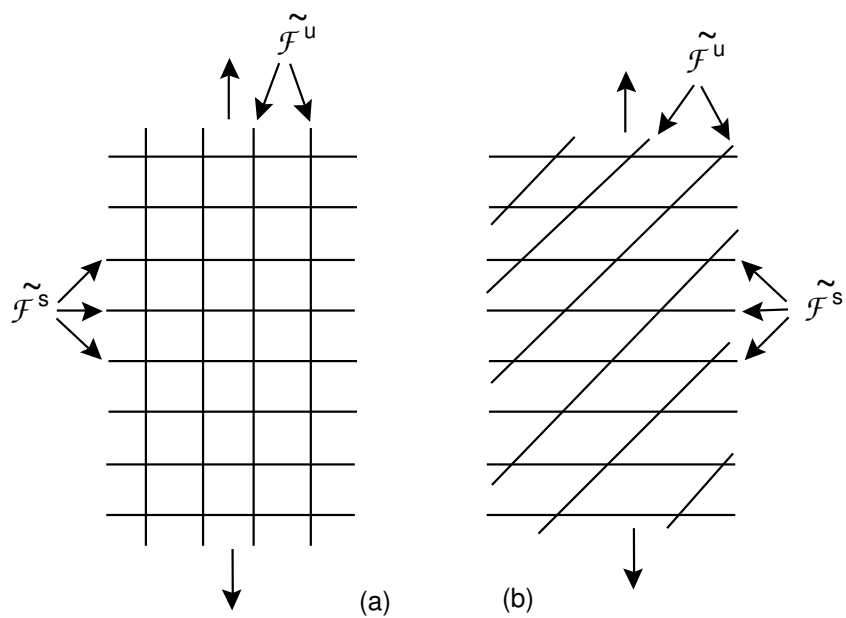

(a)

(b)

Figure 1. a. Product type. b. Skewed type. The diagrams are bounded in the horizontal direction and unbounded in the vertical direction. 
The set $\mathcal{O}$ is homeomorphic to $H=(0,1) \times \mathbf{R}$, which is more convenient for our description of $\mathbf{R}$-covered Anosov flows. In the product type the foliation $\widetilde{\mathcal{F}}^{s}$ is a foliation by horizontal segments in $H$ and $\widetilde{\mathcal{F}}^{u}$ is a foliation by vertical lines. Notice that any leaf $F$ of $\widetilde{\mathcal{F}}^{s}$ intersects every leaf of $\widetilde{\mathcal{F}}^{u}$ and vice versa.

In the skewed model $\widetilde{\mathcal{F}}^{s}$ is again a foliation by horizontal segments in $H$ and now $\widetilde{\mathcal{F}}^{u}$ is a foliation by parallel segments in $H$ making an angle $\theta \neq \pi / 2$ with the horizontal. Hence any leaf of $\widetilde{\mathcal{F}}^{s}$ does not intersect all leaves of $\widetilde{\mathcal{F}}^{u}$ and vice versa.

We now analyse the skewed type in more detail. Since $\widetilde{M}$ is simply connected $\widetilde{\mathcal{F}}^{s}, \widetilde{\mathcal{F}}^{u}$ are transversely orientable and we fix a transversal orientation. For any leaf $G \in \widetilde{\mathcal{F}}^{u}$ let $F^{\prime} \in \widetilde{\mathcal{F}}^{s}$ with $F^{\prime} \cap G \neq \emptyset$. Then there is a unique leaf $F \in \widetilde{\mathcal{F}}^{s}$ in the positive side of $F^{\prime}$, so that $F \cap G=\emptyset$, but for any stable leaf $E$ between $F$ and $F^{\prime}$ it follows that $E \cap G \neq \emptyset$. This strongly uses the fact that $\Phi$ is $\mathbf{R}$-covered (so that between $F$ and $E$ always makes sense) and of skewed type (to find $F$ with $F \cap G=\emptyset$ ). It is easy to see that $F$ depends only on $G$. We then say that $G$ is asymptotic to $F$ in the positive direction (transversally to $\widetilde{\mathcal{F}}^{s}$ ). This defines a map

$$
\Theta_{+}^{u}: \mathcal{H}^{u} \rightarrow \mathcal{H}^{s}, \quad \Theta_{+}^{u}(G)=F,
$$

which, by the description of the skewed structure, is easily seen to be a homeomorphism. In the same way the asymptotic stable leaf to $G$ in the negative direction defines $\Theta_{-}^{u}(G)$. An analogous construction defines: $\Theta_{+}^{s}, \Theta_{-}^{s}: \mathcal{H}^{s} \rightarrow \mathcal{H}^{u}$.

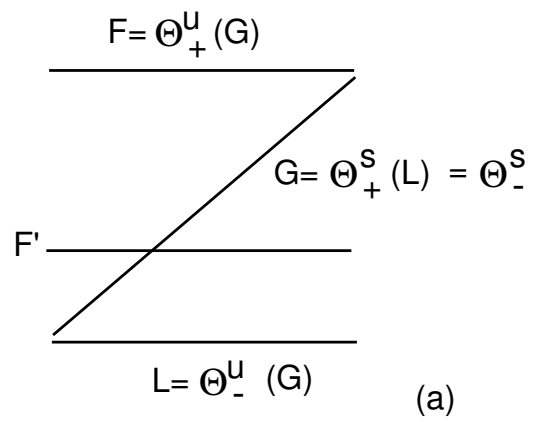

Figure 2. a. Leaf space homeomorphisms. b. Intrinsic ideal points of a stable leaf.

Choose the transversal orientation to $\widetilde{\mathcal{F}}^{u}$ so that

$$
\Theta_{+}^{s}=\left(\Theta_{-}^{u}\right)^{-1} \text { and } \Theta_{-}^{s}=\left(\Theta_{+}^{u}\right)^{-1},
$$


see Fig. 2a. This description will be essential for all the results in this article.

Suspensions have product type and geodesic flows have skewed type.

Given a leaf $F$ of $\widetilde{\mathcal{F}}^{s}$, it is topologically a product of flow lines with the strong stable direction. Ideal points of $F$ correspond to all negative limit points of flow lines in $F$ and the common positive ideal point, which is denoted by $F_{+}[\mathbf{F e} 3]$ see Fig. 2b. Geometrically, $F$ resembles the hyperbolic plane $\mathbf{H}^{2}$ where the flow lines correspond to geodesics in $\mathbf{H}^{2}$ having a distinguished ideal point in the circle at infinity of $\mathbf{H}^{2}$. If $G \in \widetilde{\mathcal{F}}^{u}$, then $G_{-} \in \partial_{\infty} G$ is the common negative point in $\partial_{\infty} G$ of all flow lines in $G$.

Given $x \in \widetilde{M}$ let $\widetilde{W}^{s}(x)$ be the leaf of $\widetilde{\mathcal{F}}^{s}$ containing $x$. Similarly define $\widetilde{W}^{u}(x)$ and $\widetilde{W}^{s}(\gamma), \widetilde{W}^{u}(\gamma)$ for $\gamma$ orbit of $\widetilde{\Phi}$.

Given $\gamma$ an orbit in $F \in \widetilde{\mathcal{F}}^{s} \cup \widetilde{\mathcal{F}}^{u}$, let $\gamma_{-} \in \partial_{\infty} F$ be its $\underline{\text { intrinsic negative }}$ limit point so that for any $x \in \gamma$ :

$$
\gamma_{-}=x_{-}=\lim _{v \rightarrow-\infty} \widetilde{\Phi}_{v}(x) \in \partial_{\infty} F
$$

where the limit is computed in $F \cup \partial_{\infty} F$. Similarly define $\gamma_{+}$and $x_{+} \in \partial_{\infty} F$.

We now explain how one can "naturally" relate points from distinct intrinsic ideal boundaries. Notice that all but one of the ideal points in $\partial_{\infty} F$, $F \in \widetilde{\mathcal{F}}^{s}$ are negative ideal points. Since flow lines in unstable leaves are asymptotic in the negative direction, negative ideal points in a stable leaf should be naturally associated to ideal points in nearby stable leaves. That is, let $p \in \partial_{\infty} F^{\prime}$ a negative ideal point and $\gamma$ a flow line in $F^{\prime}$ with $\gamma_{-}=p$. Then for sufficiently nearby $F \in \widetilde{\mathcal{F}}^{s}$, it follows that $\widetilde{W}^{u}(\gamma) \cap F \neq \emptyset$ and this intersection is an orbit $\alpha$ in $F$. If $q=\gamma_{-}^{\prime} \in \partial_{\infty} F$, then $p$ should be associated to $p^{\prime}$. If $\Phi$ is $\mathbf{R}$-covered and skewed, this approach does not work for all ideal points of $\partial_{\infty} F^{\prime}$ as we now explain: We will again refer to Fig. 2a. Fix a leaf $F^{\prime} \in \widetilde{\mathcal{F}}^{s}$ and fix a nearby $F \in \widetilde{\mathcal{F}}^{s}$ in the positive side of $F^{\prime}$. Then as seen in Fig. 2a, there are unstable leaves intersecting $F^{\prime}$ which do not intersect $F$ (corresponding to those in the back side of $G$ ). This shows that the association of ideal points of flow lines in the same unstable leaf cannot be carried out for all ideal points in $\partial_{\infty} F^{\prime}$. In order to produce a homeomorphism between the entire boundaries $\partial_{\infty} F^{\prime}$ and $\partial_{\infty} F$ we will strongly use the $\mathbf{R}$-covered hypothesis. We stress that in the skewed case not all ideal points will be mapped to ideal points of asymptotic flow lines in the same unstable leaf.

We now prove Theorem A of the introduction.

Theorem 2.1. Let $\Phi$ be an $\mathbf{R}$-covered Anosov flow. Then for any $F, L \in \widetilde{\mathcal{F}}^{s}$ there is a canonical homeomorphism $X_{F}^{L}: \partial_{\infty} F \rightarrow \partial_{\infty} L$. In addition if $E \in \widetilde{\mathcal{F}}^{s}$ then $X_{F}^{E}=X_{L}^{E} \circ X_{F}^{L}$. This defines the stable ideal boundary $S_{\infty}^{s}$ of $\Phi$ as the quotient of $\cup_{F \in \widetilde{\mathcal{F}}^{s}} \partial_{\infty} F$, by the homeomorphisms above. Then 
for any $E \in \widetilde{\mathcal{F}}^{s}$ there is homeomorphism $\zeta_{E}: \partial_{\infty} E \rightarrow S_{\infty}^{s}$ so that for any $F, L \in \widetilde{\mathcal{F}}^{s}$, it follows that $\zeta_{F}=\zeta_{L} \circ X_{F}^{L}$.

Proof. Case 1: $\Phi$ has product type.

For any $F, L \in \widetilde{\mathcal{F}}^{s}$ and any negative limit point $p$ of $F$, there is a unique flow line $\gamma$ in $F$ with $\gamma_{-}=p$. By the product description there is a unique flow line $\beta$ in $L$ so that $\gamma$ and $\beta$ are in the same unstable leaf of $\widetilde{\mathcal{F}}^{u}$. Let $X_{F}^{L}(p)=\beta_{-} \in \partial_{\infty} L$. Let $X_{F}^{L}\left(F_{+}\right)=L_{+}$.

Case 2: $\Phi$ has skewed type.

Let $p \in \partial_{\infty} F$ and assume that $L$ is in the positive side of $F$. Assume first that $p$ is not $F_{+}$. Then there is a unique orbit $\gamma$ in $F$ with $\gamma_{-}=p$. Let $G_{0}=\widetilde{W}^{u}(\gamma)$. If $G_{0} \cap L \neq \emptyset$, then $G_{0} \cap L$ is a flow line in $L$ and let

$$
X_{F}^{L}(p)=\left(G_{0} \cap L\right)_{-} \in \partial_{\infty} L .
$$

If $L=\Theta_{+}^{u}(G)$, then let $X_{F}^{L}(p)=L_{+}$.

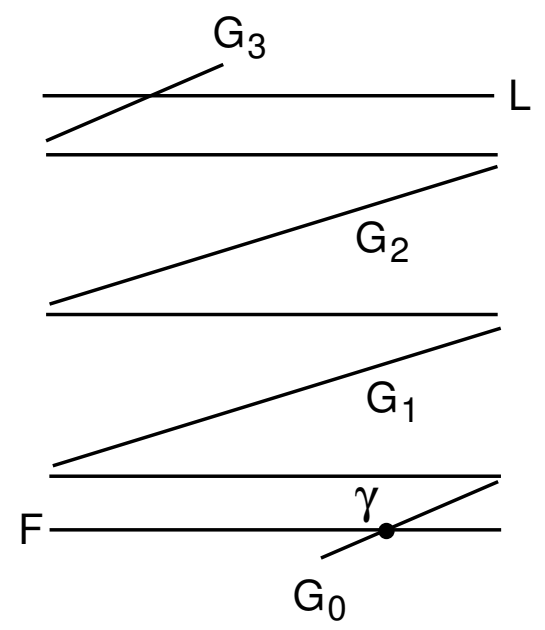

Figure 3. Identifications of intrinsic ideal points.

In the other cases let $G_{1}=\left(\Theta_{-}^{u}\right)^{-1} \circ \Theta_{+}^{u}(G)$. If $G_{1} \cap L \neq \emptyset$, let

$$
X_{F}^{L}(p)=\left(G_{1} \cap L\right)_{-} .
$$

If $L=\Theta_{+}^{u}\left(G_{1}\right)$ then $X_{F}^{L}(p)=L_{+}$. By the description of the skewed structure one eventually arrives at a leaf $G_{i}$ which either intersects $L$ or is asymptotic to $L$. The construction of the map is finished by induction. The case $p=F_{+}$ is treated similarly.

In both cases it is easy to check that for any $F, L, E \in \widetilde{\mathcal{F}}^{s}$ then

$$
X_{L}^{E} \circ X_{F}^{L}=X_{F}^{E}
$$


Hence the idenfications are well defined. The space $S_{\infty}^{s}$ is the quotient of $\cup_{F \in \widetilde{\mathcal{F}}^{s}} \partial_{\infty} F$ by the homeomorphisms between distinct intrinsic ideal boundaries. Then $S_{\infty}^{s}$ is homeomorphic to a circle and there is a well defined homeomorphism $\zeta_{F}: \partial_{\infty} F \rightarrow S_{\infty}^{s}$ for any $F \in \widetilde{\mathcal{F}}^{u}$, satisfying $\zeta_{F}=\zeta_{L} \circ X_{F}^{L}$.

In the skewed case there is an equivalent description of $S_{\infty}^{s}, S_{\infty}^{u}$ using only the structure of $\widetilde{\mathcal{F}}^{s}, \widetilde{\mathcal{F}}^{u}$ in $\widetilde{M}$, which is given by the $\Theta$ maps. The composition

$$
\chi^{u}:\left(\Theta_{-}^{u}\right)^{-1} \circ \Theta_{+}^{u}: \mathcal{H}^{u} \rightarrow \mathcal{H}^{u}
$$

is an orientation preserving homeomorphism of $\mathcal{H}^{u}$ without fixed points and the quotient $\mathcal{H}^{u} / \chi^{u}$ is a topological space $V^{u}$ which is homeomorphic to a circle. Let $\pi^{u}: \mathcal{H}^{u} \rightarrow V^{u}$ be the projection map. Similarly $\chi^{s}:\left(\Theta_{-}^{s}\right)^{-1} \circ \Theta_{+}^{s}$ : $\mathcal{H}^{s} \rightarrow \mathcal{H}^{s}$ yields $V^{s}$ and $\pi^{s}$.

Proposition 2.2. There are natural homeomorphisms $\xi^{s}: S_{\infty}^{s} \rightarrow V^{u}$ and $\xi^{u}: S_{\infty}^{u} \rightarrow V^{s}$.

Proof. Let $F \in \widetilde{\mathcal{F}}^{s}$. Given $p \in S_{\infty}^{s}$ let $p^{\prime}=\left(\zeta_{F}\right)^{-1}(p) \in \partial_{\infty} F$. If $p^{\prime} \neq F_{+}$ then $p^{\prime}=\gamma_{-}, \gamma$ flow line in $F$. Then set

$$
\xi^{s}(p)=\xi^{s}\left(\zeta_{F}\left(p^{\prime}\right)\right)=\pi^{u}\left(\widetilde{W}^{u}(\gamma)\right) .
$$

If $p^{\prime}=F_{+}$then $\Theta_{+}^{s}(F) \in \widetilde{\mathcal{F}}^{u}$ and then let

$$
\xi^{s}(p)=\xi^{s}\left(\zeta_{F}\left(p^{\prime}\right)\right)=\pi^{u}\left(\Theta_{+}^{s}(F)\right) .
$$

The proof that $\xi^{s}$ is independent of the leaf $F$ of $\widetilde{\mathcal{F}}^{s}$ is exactly the same as the proof of Theorem 2.1. Similarly for $\xi^{u}$.

Lemma 2.3. If $g$ is a covering translation of $\widetilde{M}$ then $g$ induces homeomorphisms in $V^{s}$ and $V^{u}$.

Proof. First notice $g$ acts by homeomorphisms in $\mathcal{O}$ and in the leaf spaces of $\widetilde{\mathcal{F}}^{s}$ and $\widetilde{\mathcal{F}}^{u}$. Therefore $g$ takes asymptotic pairs of leaves to asymptotic leaves. If $g$ preserves transversal orientations then:

$$
\Theta_{+}^{u} \circ g(G)=g \circ \Theta_{+}^{u}(G), \quad \forall G \in \widetilde{\mathcal{F}}^{u} .
$$

Similarly $g$ commutes with $\Theta_{-}^{u}, \Theta_{+}^{s}, \Theta_{-}^{s}$. Hence $g$ induces orientation preserving homeomorphisms in $V^{s}$ and $V^{u}$.

If $g$ reverses orientations to either $\widetilde{\mathcal{F}}^{s}$ or $\widetilde{\mathcal{F}}^{u}$ then by the $\mathbf{R}$-covered property it follows that it reverses both transversal orientations. Therefore

$$
g \circ \Theta_{+}^{u}(G)=\Theta_{-}^{u} \circ g(G) \quad \forall G \in \widetilde{\mathcal{F}}^{u}
$$

and then $g$ induces orientation reversing homeomorphisms in $V^{s}$ and $V^{u}$. 
Corollary 2.4. If $\Phi$ is $\mathbf{R}$-covered and of skewed type, there is a natural action of $\pi_{1}(M)$ into the 2 dimensional torus $T^{2}=V^{s} \times V^{u}$.

For instance if $g$ is associated to a periodic orbit of $\Phi$ then the corresponding homeomorphism of $T^{2}$ has 4 fixed points, one of which is attracting, one repelling and the other two of hyperbolic type.

If $g$ is a covering translation of $\widetilde{M}$, then it induces an homeomorphism $g_{F}^{L}$, between $\partial_{\infty} F$ and $\partial_{\infty} L$, for any $F \in \widetilde{\mathcal{F}}^{s}$ and $L=g(F)$ as follows: If $p=\gamma_{-} \in \partial_{\infty} F$, let $g_{F}^{L}(p)=(g(\gamma))_{-} \in \partial_{\infty} L$ and let $g_{F}^{L}\left(F_{+}\right)=L_{+}$. Using Lemma 2.3 and Proposition 2.2 one obtains the following result.

Corollary 2.5. Any covering translation $g \in \pi_{1}(M)$ induces a homeomorphism $g_{s}$ of $S_{\infty}^{s}$. This means the following: Fix $p \in S_{\infty}^{s}$. Let now $F \in \widetilde{\mathcal{F}}^{s}$ be an arbitrary leaf and $L=g(F)$. Then $\zeta_{L} \circ g_{F}^{L} \circ \zeta_{F}{ }^{-1}(p)$ depends only on $p$ and not on $F$. This induces an action of $\pi_{1}(M)$ in $S_{\infty}^{s}$.

Remarks. 1) If $\Phi$ is the geodesic flow in the unit tangent bundle $T_{1} N^{2}$ of a closed surface of constant curvature -1 (for simplicity), then a stable leaf $F$ of $\widetilde{\mathcal{F}}^{s}$ is isometric to the hyperbolic plane $\mathbf{H}^{2}$. The positive limit point of $F$ is just the positive limit point of the geodesic of $\mathbf{H}^{2}$ through any $x \in F(x$ is a point in $\mathbf{H}^{2}$ with a unit tangent vector). The homeomorphism between ideal boundaries in Case 2 of Theorem 2.1 is just the identity map in the circle at infinity.

2) In general one should think of the the stable ideal boundary $S_{\infty}^{s}$ as being "rotated" by moving transversally to leaves of $\widetilde{\mathcal{F}}^{s}$. Rotation means that the distinguished positive ideal point is moving around $S_{\infty}^{s}$ and returns to its original position after a " $2 \pi$ " turn, that is, after applying $\left(\Theta_{-}^{s}\right)^{-1} \circ \Theta_{+}^{s}$. This is exactly what happens for the geodesic flow.

\section{Equivariant continuous extension.}

From now on assume that $M^{3}$ is ncl. Let $\Phi$ be an Anosov flow in $M$. As proved in $[\mathbf{F e} 3], \Phi$ cannot have product type and is therefore of skewed type. This fact will be implicitly used from now on. Assume in addition that $\Phi$ has CEP. Then we can define two maps $\eta_{-}, \eta_{+}: \widetilde{M} \rightarrow S_{\infty}^{2}$ by

$$
\eta_{-}(p)=\lim _{v \rightarrow-\infty} \widetilde{\Phi}_{v}(p) \in S_{\infty}^{2} .
$$

The same ideal point is obtained for all points in the orbit $\gamma$ of $\widetilde{\Phi}$ through $p$ so we will also use the notation $\eta_{-}(\gamma)$. Similarly define $\eta_{+}$.

As a consequence of the detailed analysis of $\mathbf{R}$-covered Anosov flows and intrinsic ideal points in the previous section, most results in this section will be straightforward.

Proposition 3.1. The maps $\eta_{+}, \eta_{-}: \widetilde{M} \rightarrow S_{\infty}^{2}$ are continuous. 
Proof. Let $x_{i} \rightarrow x$ in $\widetilde{M}$. By the local product structure of $\widetilde{\Phi}$, there are $y_{i} \in \widetilde{W}^{s}\left(x_{i}\right)$ with $y_{i} \in \widetilde{W}^{u u}(x)$ (for $i \mathrm{big}$ ) and $y_{i} \rightarrow x$. Then $\eta_{+}\left(y_{i}\right)=\eta_{+}\left(x_{i}\right)$. Let $G=\widetilde{W}^{u}(x)$. In the intrinsic structure of $G$ the flow lines through $y_{i}$ are converging to that through $x$, which means that $\left(y_{i}\right)_{+} \rightarrow x_{+}$in $\partial_{\infty} G$. By the continuous extension of $\varphi_{G}: G \rightarrow \widetilde{M}$ to $\varphi_{G}: G \cup \partial_{\infty} G \rightarrow \widetilde{M} \cup S_{\infty}^{2}$, it follows that

$$
\eta_{+}\left(x_{i}\right)=\eta_{+}\left(y_{i}\right)=\varphi_{G}^{\infty}\left(\left(y_{i}\right)_{+}\right) \rightarrow \varphi_{G}^{\infty}\left(x_{+}\right)=\eta_{+}(x), \text { as } i \rightarrow \infty .
$$

Lemma 3.2. If $F \in \widetilde{\mathcal{F}}^{s}, G \in \widetilde{\mathcal{F}}^{u}$ are asymptotic, that is, either $F=\Theta_{+}^{u}(G)$ or $F=\Theta_{-}^{u}(G)$, then $\varphi_{F}^{\infty}\left(F_{+}\right)=\varphi_{G}^{\infty}\left(G_{-}\right)$.

Proof. Suppose $F=\Theta_{+}^{u}(G)$, the other case being similar. Let $E \in \widetilde{\mathcal{F}}^{s}$ with $E \cap G \neq \emptyset$ and let $E \cap G=\gamma$, orbit of $\widetilde{\Phi}$. Let $\gamma_{i} \subset E, \gamma_{i}$ orbits of $\widetilde{\Phi}$ with $\widetilde{W}^{u}\left(\gamma_{i}\right) \cap F \neq \emptyset$ and $\gamma_{i} \rightarrow \gamma$ in $E$, see Fig. 4. Let $\beta_{i}=\widetilde{W}^{u}\left(\gamma_{i}\right) \cap F$.

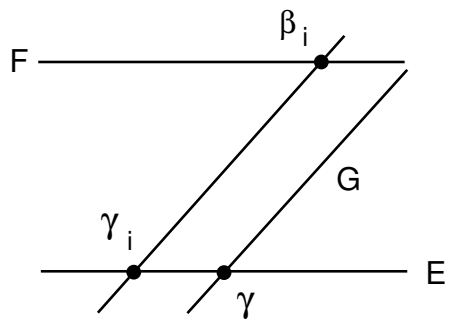

Figure 4. Equal ideal points.

Then the sequence $\left\{\beta_{i}\right\}_{i \in \mathbf{N}}$ escapes to infinity in $F$ as $i \rightarrow \infty$ and hence $\left(\beta_{i}\right)_{-} \rightarrow F_{+}$in $\partial_{\infty} F$ as $i \rightarrow \infty$. By CEP, $\eta_{-}\left(\beta_{i}\right)=\varphi_{F}^{\infty}\left(\left(\beta_{i}\right)_{-}\right) \rightarrow \varphi_{F}^{\infty}\left(F_{+}\right)$. Since $\beta_{i} \subset \widetilde{W}^{u}\left(\gamma_{i}\right), \eta_{-}\left(\beta_{i}\right)=\eta_{-}\left(\gamma_{i}\right)$, hence

$$
\varphi_{G}^{\infty}\left(G_{-}\right)=\eta_{-}(\gamma)=\lim _{i \rightarrow \infty} \eta_{-}\left(\gamma_{i}\right)=\lim _{i \rightarrow \infty} \eta_{-}\left(\beta_{i}\right)=\varphi_{F}^{\infty}\left(F_{+}\right)
$$

We now prove Theorem B of the introduction.

Theorem 3.3. Let $\Phi$ be an $\mathbf{R}$-covered Anosov flow in $M^{3}$ ncl and assume that $\Phi$ has $C E P$. Then there is a continuous map $\varphi_{s}: S_{\infty}^{s} \rightarrow S_{\infty}^{2}$ so that for any $F \in \widetilde{\mathcal{F}}^{s}, \varphi_{F}^{\infty}=\varphi_{s} \circ \zeta_{F}$.

Proof. Let $q \in S_{\infty}^{s}$ and let $p \in \partial_{\infty} R, R \in \widetilde{\mathcal{F}}^{s}$, be a representative of $q$. If $p=$ $R_{+}$let $F=R, G=\Theta_{+}^{s}(F)$ and $L=\Theta_{+}^{u}(G)$. Otherwise let $p=\gamma_{-}$, where $\gamma$ is a flow line in $R$. Then let $G=\widetilde{W}^{u}(\gamma), F=\Theta_{-}^{u}(G)$ and $L=\Theta_{+}^{u}(G)$. 
By the description of Proposition 2.2, it suffices to show that if $p_{E}$ is a representative of $q$ in $E \in \widetilde{\mathcal{F}}^{s}$, where $E$ is between $F$ and $L$, then $\varphi_{E}^{\infty}\left(p_{E}\right)$ is independent of the particular leaf $E$. If $E=F$, then

$$
p_{E}=F_{+} \quad \text { and } \quad \varphi_{F}^{\infty}\left(p_{E}\right)=\varphi_{F}^{\infty}\left(F_{+}\right) .
$$

If $E \neq F, L$, then $p_{E}=\left(\gamma_{E}\right)_{-} \in \partial_{\infty} E$, where $\gamma_{E}=E \cap G$. As $\gamma_{E} \subset G$, then

$$
\varphi_{E}^{\infty}\left(p_{E}\right)=\varphi_{E}^{\infty}\left(\left(\gamma_{E}\right)_{-}\right)=\eta_{-}\left(\gamma_{E}\right)=\varphi_{G}^{\infty}\left(G_{-}\right) .
$$

If $E=L$, then $p_{E}=L_{+}$and as in the first case, $\varphi_{L}^{\infty}\left(p_{E}\right)=\varphi_{L}^{\infty}\left(L_{+}\right)$. By the previous lemma $\varphi_{F}^{\infty}\left(F_{+}\right)=\varphi_{G}^{\infty}\left(G_{-}\right)=\varphi_{L}^{\infty}\left(L_{+}\right)$, which finishes the proof.

We now have various maps associated to a given covering translation $g$ of $\widetilde{M}$, all of which are homeomorphisms:

- $g_{F}^{L}: \partial_{\infty} F \rightarrow \partial_{\infty} L$, where $F \in \widetilde{\mathcal{F}}^{s}$ and $L=g(F)$,

- $g_{F}=g_{F}^{F}$, if $g(F)=F$,

- $g_{\infty}: S_{\infty}^{2} \rightarrow S_{\infty}^{2}$, continuous extension of $g: \widetilde{M} \rightarrow \widetilde{M}$, which exists for any $M^{3} \mathrm{ncl}[\mathbf{G r}]$ and

- $g_{s}: S_{\infty}^{s} \rightarrow S_{\infty}^{s}, \quad g_{u}: S_{\infty}^{u} \rightarrow S_{\infty}^{u}$.

Using Theorem 3.3, together with Lemma 2.3 and Proposition 2.2 it immediately follows that:

Corollary 3.4. The extension $\varphi_{s}: S_{\infty}^{s} \rightarrow S_{\infty}^{2}$ is invariant under covering translations, that is, for any covering translation $g$ of $\widetilde{M}, g_{\infty} \circ \varphi_{s}=\varphi_{s} \circ g_{s}$ and similarly for the unstable foliation.

We now prove Theorem C:

Proposition 3.5. Let $\Phi$ be an $\mathbf{R}$-covered Anosov flow in $M^{3}$ ncl and assume that $\Phi$ has $C E P$. For any $A$ any open in $S_{\infty}^{s}, \varphi_{s}(A)$ contains an open set in $S_{\infty}^{2}$.

Proof. Since $\Phi$ is transitive, the periodic orbits are dense in $\mathcal{O}[\mathbf{S m}]$. Notice that $A$ is homeomorphic to an open set $B \subset \partial_{\infty} F, F \in \widetilde{\mathcal{F}}^{s}$, where there is a periodic orbit $\gamma$ in $F$ with $p=\gamma_{-} \in B$. Let $g$ be the covering translation associated to the closed orbit $\pi(\gamma)$ of $\Phi$ so that $g(F)=F$. By Corollaries 2.5 and 3.4, $g_{\infty} \circ \varphi_{F}^{\infty}=\varphi_{F}^{\infty} \circ g_{F}$. Recall that $g_{F}$ fixes 2 points $p$ and $F_{+} \in \partial_{\infty} F$ and we may assume $p$ is the expanding fixed point. For $n>0$ big enough, $\left(\partial_{\infty} F-g_{F}^{n}(B)\right)$ is contained in a small enough neighborhood of $F_{+}$. By continuity of $\varphi_{F}^{\infty}$ it follows that $\varphi_{F}^{\infty}\left(\partial_{\infty} F-g^{n}(B)\right)$ is contained in a small neighborhood of $\varphi_{F}^{\infty}\left(F_{+}\right)$. Therefore $\varphi_{F}^{\infty}\left(g_{F}^{n}(B)\right)$ contains an open neighborhood of $\varphi_{F}^{\infty}(p)$. Since $g_{\infty}$ fixes $\varphi_{F}^{\infty}(p)$ it follows that $\varphi_{F}^{\infty}(B)=$ $g_{\infty}^{-n}\left(\varphi_{F}^{\infty}\left(g_{F}^{n}(B)\right)\right)$ will contain an open neighborhood of $\varphi_{F}^{\infty}(p)$ as desired. 


\section{Non injectivity of the continuous extension.}

Proposition 4.1. Let $\Phi$ be an $\mathbf{R}$-covered Anosov flow in $M^{3}$ ncl and assume that $\Phi$ has CEP. Let $F$ be a periodic leaf of $\widetilde{\mathcal{F}}^{s}$ with $\gamma$ the periodic orbit in F. Let $p=\gamma_{-}$. If $\varphi_{F}^{\infty}\left(F_{+}\right)=\varphi_{F}^{\infty}(z)$ for $z \in \partial_{\infty} F$ then $z=F_{+}$and similarly for $p$.

Proof. Suppose that $\varphi_{F}^{\infty}\left(F_{+}\right)=\varphi_{F}^{\infty}(z)$. If $z=p$ then $\gamma$ is an orbit of $\widetilde{\Phi}$ with same ideal points in the forward and backward directions. But $\pi(\gamma)$ is a closed orbit of $\Phi$ in $M$, so it is freely homotopic to a closed geodesic of $M$ $[\mathbf{G r}]$. Hence $\gamma$ is a quasigeodesic in $\widetilde{M}$ and it cannot have identified ideal points $[\mathbf{G r}]$.

Let $g$ be a non trivial covering translation of $\widetilde{M}$ with $g(F)=F$ and assume that $p$ is the attracting fixed point. If $\varphi_{F}^{\infty}\left(F_{+}\right)=\varphi_{F}^{\infty}(w)$ with $w \neq F_{+}, p$, then $g_{F}^{n}(w) \rightarrow p\left(\right.$ in $\left.\partial_{\infty} F\right)$ as $n \rightarrow+\infty$, so

$$
\varphi_{F}^{\infty}\left(F_{+}\right)=g_{\infty}^{n}\left(\varphi_{F}^{\infty}\left(F_{+}\right)\right)=g_{\infty}^{n}\left(\varphi_{F}^{\infty}(w)\right)=\varphi_{F}^{\infty}\left(g_{F}^{n}(w)\right) \rightarrow \varphi_{F}^{\infty}(p) .
$$

Hence $\varphi_{F}^{\infty}\left(F_{+}\right)=\varphi_{F}^{\infty}(p)$, contradiction to the above. Similarly for $p$.

We say that $p \in S_{\infty}^{s}$ is in the class of a periodic stable point, if there is $F \in \widetilde{\mathcal{F}}^{s}$ periodic and $\gamma$ the periodic orbit in $F$, so that either $p=\zeta_{F}\left(\gamma_{-}\right)$or $p=\zeta_{F}\left(\gamma_{+}\right)$. We now prove Theorem D:

Theorem 4.2. Let $\Phi$ be an $\mathbf{R}$-covered Anosov flow in $M^{3}$ ncl and assume that $\Phi$ has CEP. If $p \in S_{\infty}^{s}$ is in the class of a periodic orbit, then $\varphi_{s}(p)=$ $\varphi_{s}(q)$ implies that $p=q$.

Proof. Let $F \in \widetilde{\mathcal{F}}^{s}$ periodic, $\gamma$ periodic orbit in $F$ and $p=\zeta_{F}\left(\gamma_{-}\right)$. Let $z=\gamma_{-} \in \partial_{\infty} F$ and let $w \in \partial_{\infty} F$ with $w=\left(\zeta_{F}\right)^{-1}(q)$. By Theorem 3.3

$$
\varphi_{F}^{\infty}(z)=\varphi_{s}(p)=\varphi_{s}(q)=\varphi_{F}^{\infty}(w) .
$$

By the previous proposition, $z=w$, hence $p=q$. Similarly if $p=\zeta_{F}\left(\gamma_{+}\right)$.

Since $\varphi_{s}\left(S_{\infty}^{s}\right)=S_{\infty}^{2}$ and $S_{\infty}^{s}$ is homeomorphic to a circle, it follows that $\varphi_{s}$ is not injective. One open question is to decide whether this map can be infinite to one. This is impossible in the quasigeodesic, non $\mathbf{R}$-covered case [Fe4]. We will prove there are uncountably many points in $S_{\infty}^{s}$ which have the same image under $\varphi_{s}$ as some other point of $S_{\infty}^{s}$.

Definition 4.3. We say that an orbit $\beta$ of $\widetilde{\Phi}$ is pinched if $\eta_{+}(\beta)=\eta_{-}(\beta)$. Since for any covering translate $g(\beta)$ of $\beta, g_{\infty}\left(\eta_{-}(\beta)\right)=\eta_{-}(g(\beta))$, this is really a property of $\pi(\beta)$. We then say $\pi(\beta)$ is liftable pinched. 
A pinched orbit is reminiscent of a horocycle in hyperbolic space. Recall that a horocycle has well defined limit points which are the same in both directions. Clearly it is not quasigeodesic.

Proposition 4.4. There is a pinched orbit of $\widetilde{\Phi}$.

Proof. Fix $F \in \widetilde{\mathcal{F}}^{s}$ and let $p \neq q \in \partial_{\infty} F$ with $\varphi_{F}^{\infty}(p)=\varphi_{F}^{\infty}(q)$. If $p$ or $q$ is $F_{+}$then we are done. Otherwise let $x, y \in F$ with $p=x_{-}, q=y_{-}$. Let $L \in \widetilde{\mathcal{F}}^{s}$, in the positive side of $F$, which is asymptotic to $\widetilde{W}^{u}(x)$ and intersects $\widetilde{W}^{u}(y)$ or vice versa. Assume $L=\Theta_{+}^{u}\left(\widetilde{W}^{u}(x)\right)$, then $L_{+}=X_{F}^{L}(p)$. Since $X_{F}^{L}(q) \neq L_{+}$, the result follows from:

$$
\varphi_{L}^{\infty}\left(L_{+}\right)=\varphi_{L}^{\infty}\left(X_{F}^{L}(p)\right)=\varphi_{F}^{\infty}(p)=\varphi_{F}^{\infty}(q)=\varphi_{L}^{\infty}\left(X_{F}^{L}(q)\right) .
$$

Proposition 4.5. If $\gamma$ is a liftable pinched orbit of $\Phi$ then any orbit in its closure $\bar{\gamma}$ is also liftable pinched.

Proof. Let $\alpha \subset \bar{\gamma}$, but not $\gamma$ itself. Let $x \in \alpha, y \in \gamma$. Then there are $v_{i} \in \mathbf{R},\left|v_{i}\right| \rightarrow+\infty$, with $\Phi_{v_{i}}(y) \rightarrow x$. Let $x^{\prime} \in \widetilde{M}$ with $\pi\left(x^{\prime}\right)=x$ and choose $y_{i} \in \pi^{-1}\left(\Phi_{v_{i}}(y)\right)$, with $y_{i} \rightarrow x^{\prime}$. Then $\eta_{-}\left(y_{i}\right) \rightarrow \eta_{-}(x)$ and similarly for the positive direction. Since $\gamma$ is liftable pinched, $\eta_{-}\left(y_{i}\right)=\eta_{+}\left(y_{i}\right)$ for all $i$. Then $\eta_{+}\left(x^{\prime}\right)=\eta_{-}\left(x^{\prime}\right)$ and $\alpha$ is liftable pinched as desired.

If $\gamma$ is a closed orbit of $\Phi$ then $\gamma$ is not null homotopic in $M$ [An]. It follows that any lift $\widetilde{\gamma}$ to $\widetilde{M}$ is a quasigeodesic. Hence $(\widetilde{\gamma})_{-} \neq(\widetilde{\gamma})_{+}$and we conclude that $\gamma$ is not liftable pinched. The following is immediate:

Corollary 4.6. If $\gamma$ is liftable pinched then $\bar{\gamma}$ does not contain a closed orbit. In particular no dense orbit of $\Phi$ is liftable pinched.

Recall that an orbit of a flow is almost periodic [Bo] if its closure is a minimal set. Bowen $[\mathbf{B o}]$ proved that non periodic, almost periodic orbits of Axiom A flows form a dense set in the non wandering set. We restate Theorem E as follows:

Theorem 4.7. Let $\Phi$ be an $\mathbf{R}$-covered Anosov flow in $M^{3}$ ncl and assume that $\Phi$ has CEP. Then there are uncountably many liftable pinched orbits of $\Phi$. In particular there are uncountably many such orbits which are almost periodic.

Proof. By Proposition 4.4 there is an orbit $\beta$ of $\Phi$ which is liftable pinched. Then $\bar{\beta}$ contains a minimal set of the flow which by Corollary 4.6 cannot be a closed orbit, hence it is the closure of an almost periodic orbit $\gamma$. By Proposition 4.5 any orbit in this closure is liftable pinched. The closure does not contain isolated orbits since it is a minimal set and not a closed orbit. Consider the intersection of $\bar{\gamma}$ with a small transversal disk to the flow $\Phi$. 
By the above this is a perfect set and therefore a Cantor set. Since any orbit intersects this disk at most countably many times, there are uncountably many orbits in $\bar{\gamma}$.

Some remarks are in order here. One may be tempted to prove a result similar to Corollary 4.6 for almost periodic orbits. For instance, using the fact that an almost periodic orbit $\gamma$ is recurrent, any return of $\gamma$ near to itself defines by the shadow lemma [Bo] a nearby closed orbit of $\Phi$, which could theoretically be used to prove that there are no liftable pinched, almost periodic orbits. One of the problems with this argument is that as $\gamma$ keeps returning near itself the sequence of closed orbits obtained by the shadow lemma gets more and more complicated, in particular their lengths go to infinity and very little information can be recovered.

As a matter of fact, almost periodic orbits do not behave "almost" like periodic orbits. Their complexity can be understood using symbolic dynamics: Bowen and Walters $[\mathbf{B o}, \mathbf{B o}-\mathbf{W a}]$ proved that the flow restricted to the closure of an almost periodic, non periodic orbit is conjugate to a variable time suspension of a subshift (not of finite type). The key fact is that general subshifts can be quite complicated, far from having the good properties that subshifts of finite type possess, in particular, the flow restricted to the closure of an almost periodic orbit can be quite complicated.

\section{References}

[An] D.V. Anosov, Geodesic flows on closed Riemannian manifolds with negative curvature, Proc. Steklov Inst. Math., 90 (1969).

[Ba1] T. Barbot, Caractérization des flots d'Anosov en dimension 3 par leurs feuilletages faibles, Erg. Th. Dyn. Sys., 15 (1995), 247-270.

[Ba2] _ Flots d'Anosov sur les variétés graphées aus sens de Waldhausen, Ann. Inst. Fourier, 46 (1996), 1451-1517.

[Be-Me] M. Bestvina and J. Mess, The boundary of negatively curved groups, Jour. Amer. Math. Soc., 4 (1991), 469-481.

[Bon] F. Bonahon, Bouts des variétés hyperboliques de dimension 3, Ann. of Math., 124 (1986), 71-158.

[Bo-La] C. Bonatti and R. Langevin, Un example de flot d'Anosov transitif transverse a un tore et non conjugue a une suspension, Erg. Th. Dyn. Sys., 14 (1994), 633-643.

[Bo] R. Bowen, Symbolic dynamics for hyperbolic flows, Amer. Jour. of Math. 95 (1973) 429-460.

[Bo-Wa] R. Bowen and P. Walters, Expansive one parameter flows, Jour. Diff. Eq., 12 (1972), 180-193.

[Can] A. Candel, Uniformization of surface laminations, Ann. Sci. L'Ecole Norm. Sup., 26 (1993), 489-516.

[Ca-Th] J. Cannon and W. Thurston Group invariant peano curves, to appear.

[Fe1] S. Fenley, Quasi-isometric foliations, Topology, 31 (1992), 667-676. 
[Fe2] - Asymptotic properties of depth one foliations in hyperbolic 3-manifolds, Jour. Diff. Geo., 36 (1992), 269-313.

[Fe3] _ Anosov flows in 3-manifolds, Ann. Math., 139 (1994), 79-115.

[Fe4] Quasigeodesic Anosov flows and homotopic properties of flow lines, Jour. Diff. Geom., 41 (1995), 479-514.

[Go] S. Goodman, Dehn surgery and Anosov flows, in Proceedings of the geometric dynamics conference, Lecture notes in mathematics 1007, Springer, 1983.

[Gr] M. Gromov, Hyperbolic groups, in Essays on group theory, 75-263, Springer, 1987.

[Sm] S. Smale, Differentiable dynamical systems, Bull. Amer. Math. Soc., 73 (1967), 747-817.

[So] V.V. Solodov, On the universal cover of Anosov flows, Research announcemment, 1991.

[Su] D. Sullivan, Cycles for the dynamical study of foliated manifolds and complex manifolds, Invent. Math., 36 (1976), 225-255.

[Th1] W. Thurston, The geometry and topology of 3-manifolds, Princeton University Lecture Notes, 1982.

[Th2] _ Hyperbolic structures on 3-manifolds II: Surface groups and 3-manifolds that fiber over the circle, preprint.

Received June 13, 1994 and revised December 30, 1996. This reseach supported by NSF grant DMS-9201744 and an NSF postdoctoral fellowship.

Princeton University

Princeton, NJ 08544-1000

E-mail address: fenley@math.princeton.edu

WASHINGTON UNIVERSITY

St. Louis, MO 63130 